Pacific Journal of Mathematics

RNGS WTIH PRoPER INOULT 


\title{
ON RINGS WITH PROPER INVOLUTION
}

\author{
W. E. BAXter
}

A topological ring is said to have property $(Y)$ if, and only if, $2 A=A$; $A$ has a proper continuous involution (with symmetric elements $S$ ) such that whenever the net $\left\{2 x_{\alpha}\right\}$ tends to zero, so also does $\left\{x_{\alpha}\right\} ; A^{3}$ is dense in $A$; and the left annihilator of a closed Jordan ideal, $U$, of $S$ is zero if, and only if, $U$ is $S$.

One shows that for such rings and for annihilator rings with the first two properties above that every closed Jordan ideal of $S$ is the intersection of $S$ with a closed two-sided ideal. Also shown is the fact that $S \circ S$ is dense in $S$.

A study is made of relations between the socle and Jordan ideals of $S$ for topological rings. Finally, a new proof of Herstein's result for $S$ in simple associative rings is given.

The purpose of this paper is twofold. The literature on rings with involution contains little about a Jordan ideal $U$ of $S$, the set of symmetric elements; in fact, Herstein's work [2] on such ideals in a simple ring is one of the few results in this area. Therefore, we attempt in the first part of this paper to make an algebraic beginning into such a study for rings with proper involution, and with the properties: $2 x=\theta$ implies $x=\theta$ and $2 A=A$.

We introduce the notion of topological rings with property $(Y)$, these being the analog of the semiprime rings of [1] with the property that for a closed proper two-sided ideal, $I, \mathscr{L}(I) \neq(\theta)$. We show that for these rings and for annihilator rings (with certain conditions on convergence) that a closed Jordan ideal, $U$, of $S$ is the intersection of $S$ with a closed two-sided ideal of $A$.

We then apply these results to (i) a reproof of Herstein's result (for simple rings with proper involution) making use of the fact that the subring generated by $S$ is $A$; (ii) showing that in the annihilator rings under discussion as well as for topological rings with property $(Y)$ that $S \circ S$ is dense in $S$ (these results are related to results on the positive cone in Banach algebras); and (iii) considering relationships between minimal Jordan ideals of $S$ and two-sided ideals of $A$, as well as relations concerning minimal idempotents and Jordan ideals.

2. Pure algebra. We define for each pair $(a, b)$ of elements of $A(a, b)_{J}=a b+b^{J} a^{J}$ and $(a, b)_{L}=a b-b^{J} a^{J}$. Let $U$ be a Jordan ideal of $S$, then 
LEMmA 1. (i) $s \in S$ implies that $t=(1 / 2 s)$ such that $2 t=s$ is in $S$; (ii) $u^{2} \in U$ for all $u \in U$; (iii) $u s u$ and sus $\in U$ for all $u \in U$, $s \in S$, and (iv) $\left(u^{2}, a\right)_{J} \in U$ for all $a \in A, u \in U$.

(i) is immediate. (ii) is immediate after the observation that $u v=v u$ where $v=1 / 2 u$. But, $[u, v]=x$ implies that $2 x=\theta$ and so the desired conclusion. Now

$$
\begin{aligned}
& u s u=u \circ(u \circ t)-u^{2} \circ t \text { and } \\
& s u s=2\left\{t \circ(t \circ u)-u \circ t^{2}\right\},
\end{aligned}
$$

yielding (iii), and if $a=s+k, s \in S, k \in K$ then

$$
\left(u^{2}, a\right)_{J}=u^{2} \circ s+u \circ[u, k]
$$

completing the argument.

Now define $B=\left\{b \mid b \in A,(b, a)_{J} \in U\right.$ for all $\left.a \in A\right\}$. The key to what follows is the following lemma.

Lemma 2. $U \circ U \subset B, B$ is a right ideal and $S B \subset B$.

$U \circ U \subset B$ by the last statement in Lemma 1 , and the fact that $u \circ v=(u+v)^{2}-u^{2}-v^{2}$.

It readily follows that $B$ is a right ideal, while $s \in S, b \in B$, and $a \in A$ implies that $((s b), a)_{J}=s \circ(b, a)_{J}+(b,-a s)_{J}$ the last assertion.

An involution, $J$, on $A$ is proper if, and only if, $x x^{J}=\theta x \in A$ implies $x=\theta$. Henceforth, our involution is proper. We note that this implies that there exists no nilpotent ideals, and thus by Herstein [2] no nil right or left ideals of bounded index of nilpotency. Thus the first part of the following lemma is immediate. The second follows since the involution is proper but we give a proof valid for semi-prime rings and independent of proper involution.

Lemma 3. If $x \in S$ and $x S x=(\theta)$ then $x=\theta$. Also, if $W$ is a Jordan ideal of $S$ and $w^{2}=\theta$ for all $w \in W$ then $W=(\theta)$.

For all $w \in W, w^{2}=\theta$ implies that $W \circ W=(\theta)$. Thus, $w \circ(w \circ s)=$ $2 w s w=\theta$ for all $s \in S$, and so by the first statement we are done.

If $C$ is a subset of $A$ then $\mathscr{L}(C)=\{b \in A \mid b c=\theta$ for all $c \in C\}$.

Lemma 4. $W=\mathscr{L}(B) \cap B \cap S=(\theta)$.

Now $w \in W$ then $s w \in B$ and $w s \in \mathscr{L}(B)$ by Lemma 2 for all $s \in S$. Also, $B$ and $\mathscr{L}(B)$ are respectively right and left ideals. Thus $w s \in B$ and $s w \in \mathscr{L}(B)$. So, $w s+s w \in W$; that is $W$ is a Jordan 
ideal of $S$ with property of Lemma 3 and so the desired conclusion.

Lemma 5. $\mathscr{L}(B) \cap U=(\theta)$.

Let $u \in \mathscr{L}(B) \cap U$ then by Lemma $1, u^{2} \in B \cap S$. Thus, $u^{2} \in W$, of Lemma 4 , or $u^{2}=\theta$ for all $u \in \mathscr{L}(B) \cap U$. Now, if $s \in S$ then $(u \circ s) b=u(s b)+s(u b)=\theta$ for all $b \in B$ and so $\mathscr{L}(B) \cap U$ is Jordan ideal of $S$ and by Lemma $3, \mathscr{L}(B) \cap U=(\theta)$. We are now in a position to prove our first theorem.

Theorem 1. $\mathscr{C}(B)=\mathscr{L}(U)=\mathscr{L}(B \cap U)$.

The following remarks are immediate. $\mathscr{L}(B) \subset \mathscr{L}(B \cap U)$ and $\mathscr{L}(U) \subset \mathscr{L}(B \cap U)$. Suppose we show that $\mathscr{L}(B) \subset \mathscr{L}(U)$ and $\mathscr{L}(B \cap U) \subset \mathscr{L}(B)$, then we see that we are done. Now, since $S B \subset B$ then $\mathscr{C}(B) S S \subset \mathscr{L}(B)$. Thus let $u \in U, x \in \mathscr{f}(B)$ then $x\left(x^{J} x\right)$ $u \in \mathscr{L}(B)$, a left ideal. Hence,

$$
u\left(x^{J} x\right)\left(x^{J} x\right) u=\left(u x^{J} x\right)\left(u x^{J} x\right)^{J} \in \mathscr{L}(B) \cap U,
$$

the latter by Lemma 1 (iii). Thus, by Lemma 5 and fact that involution is proper we conclude that $u x^{J} x=\theta$ or $(x u)^{J}(x u)=\theta$ for each $x \in \mathscr{L}(B), u \in U$. Thus, $x u=\theta$ and so $\mathscr{L}(B) \subset \mathscr{L}(U)$. Now let $x \in \mathscr{L}(B \cap U)$ and $b \in B$, observing that $\left(b, 1 / 2 b^{J}\right)_{J}=b b^{J} \in U$, we conclude that $x b b^{J}=\theta$. Thus, $(x b)(x b)^{J}=(\theta)$ and so $x \in \mathscr{L}(B)$, finishing the argument.

Now let $u \in B \cap U, y \in \mathscr{L}(B \cap U), k \in K$. Then

$$
(y k) u=y(u,-k)_{J}+y u k \text {. }
$$

As $u \in B$ then $(u,-k)_{J} \in U$ and so, by Theorem 1 , we conclude that $y k \in \mathscr{L}(B \cap U)$. Therefore, we have proved the first statement in the following.

Theorem 2. $\mathscr{C}(B)$ is a two-sided ideal of $A$ and $(B) \cap B=(\theta)$.

To see that $\mathscr{L}(B) \cap B=(\theta)$ all we need note is that $\mathscr{L}(B) \cap B$ is a right ideal with the property that $x^{2}=\theta$ for all $x \in \mathscr{L}(B) \cap B$.

3. Annihilator rings. Up to this point everything that has been done, has been done algebraically. Henceforth we assume that $A$ is also a topological annihilator ring with continuous involution and the property that if $\left\{2 x_{\alpha}\right\}$ is a net convergent to $\theta \in A$ then $\left\{x_{\alpha}\right\}$ is also a net converging to $\theta$. This latter remark guarantees that if $\left\{a_{\alpha}=s_{\alpha}+k_{\alpha} \mid s_{\alpha} \in S, k_{\alpha} \in K\right\}$ converges to an element in $S$ then $\left\{s_{\alpha}\right\}$ 
also converges to that element, and that if $C \leqq S, B \subseteq K$ and $C+B$ is dense in $A$ then $C$ is dense in $S$ and $B$ is dense in $K$.

The definition of an annihilator ring says that for any closed right (left) ideal $R,(L)$ of $A, \mathscr{L}(R)=(\theta)(\mathscr{R}(L)=(\theta))$ if, and only if, $R=A(L=A)$.

Now Theorem 2 has shown that $\mathscr{L}(B)+B=H$ is a right ideal and it is immediate that $x \in \mathscr{L}(H)$ implies $x^{2}=\theta$, and so by our previous reasoning with regards to nil left ideals we have that $H$ is dense in $A$.

We make good use of the following lemma.

Lemma 6. If $U$ is a closed Jordan ideal of $S$ then $B$ is a closed right ideal in $A$.

Let $b_{\alpha}$ be a net in $B, b_{\alpha} \rightarrow b$. Then $\left(b_{\alpha}-b, a\right)_{J}=\left(b_{\alpha}, a\right)_{J}-(b, a)_{J} \cdot$ Thus

$$
\left(b_{\alpha}, a\right)_{J} \longrightarrow(b, a)_{J}
$$

Each $\left(b_{\alpha}, a\right)_{J} \in U$, and $U$ is closed. Thus, $(b, a)_{J} \in U$ or $b \in B$. Henceforth, in this section $U$ is a closed Jordan ideal in $S$.

Theorem 3. $B$ is a two-sided ideal of $A$.

$H=B \oplus \mathscr{L}(B)$ is dense in $A$. Thus given $a \in A$ there exists a net $a_{\alpha} \in H$ such that $a_{\alpha} \rightarrow a$. Write $a_{\alpha}=b_{\alpha}+d_{\alpha}, b_{\alpha} \in B, d_{\alpha} \in \mathscr{L}(B)$ then for any $b \in B$,

$$
a_{\alpha} b \longrightarrow a b \text {. }
$$

But $a_{\alpha} b=b_{\alpha} b+d_{\alpha} b=b_{\alpha} b \in B$. Thus, $b_{\alpha} b=a_{\alpha} b \rightarrow a b$ and the latter is in $B$, since $B$ is closed.

Now $B$ is a two-sided ideal and so $B^{J}$ is two-sided also. Thus, $\mathscr{L}\left(B^{J}\right)=\mathscr{R}\left(B^{J}\right)$ and similarly for $B$. Thus the following is immediate.

LEMMA 7. $\mathscr{L}\left(B^{J}\right)=(\mathscr{L}(B))^{J}$.

Thus, $H^{J}=(B+\mathscr{L}(B))^{J}=B^{J}+\mathscr{L}\left(B^{J}\right)$ is dense in $A$.

Theorem 4. $B=B^{J}$ and $U \subset B$.

Let $a_{\alpha} \rightarrow a, a_{\alpha}$ a net in $H^{J}$. Then, $a_{\alpha}=b_{\alpha}^{J}+d_{\alpha}^{J}$ where $b_{\alpha}^{J} \in B^{J}$ and $d_{\alpha}^{J}=\mathscr{L}\left(B^{J}\right)$. Thus, $b_{\alpha} \in B$ and $d_{\alpha} \in \mathscr{L}(B)$ (the latter by using Lemma 7). Let $b^{J} \in B^{J}$ then $\left(b^{J}, a_{\alpha}\right)_{J}=\left(b^{J}, b_{\alpha}^{J}+d_{\alpha}^{J}\right)_{J}=\left(b^{J}, b_{\alpha}^{J}\right)_{J} \in U$. Therefore, $\left(b^{J}, a_{\alpha}\right)_{J} \rightarrow\left(b^{J}, a\right)_{J} \in U$, as $U$ is closed, or $b^{J} \in B$.

Also, for any $u \in U,\left(u, a_{\alpha}\right)_{J}=\left(b_{\alpha}, u\right)_{J}+\left(d_{\alpha}, u\right)_{J}$ as above. But 
$d_{\alpha} \in \mathscr{L}(B)=\mathscr{L}(U)$ and so $\left(u, a_{\alpha}\right)_{J}=\left(b_{\alpha}, u\right)_{J} \in U$. As before, this means that $(u, a)_{J} \in U$ for arbitrary $a \in A$, or $U \subset B$.

Theorem 5. $U=C \cap S$ for $C$ a closed two-sided ideal of $A$, and a sufficient condition for $U=B \cap S$ is that $B A$ be dense in $B$.

Let $V$ be the additive group generated by $\left\{(u, a)_{L} \mid u \in U, a \in A\right\}$. Using $U \subset B$ observe that for all $a, c \in A$ and $u \in U$ :

$$
\begin{aligned}
& a u c+c^{J} u a^{J}=\left((a, u)_{J}, c\right)_{J}+\left(u,\left(-a^{J} c\right)\right)_{J} \in U \text { and } \\
& a u c-c^{J} u a^{J}=\left((a, u)_{J}, c\right)_{L}+\left(u,\left(-a^{J} c\right)\right)_{L} \in V .
\end{aligned}
$$

Thus, $u \in U, a, c \in A$ then $u(2 c)=(u, c)_{J}+(u, c)_{L}$ or $U A \subset U+V$. Also,

$$
\begin{aligned}
(u, a)_{L}(2 c)=(u, a c)_{L}+(u, a c)_{J} & +\left\{a^{J} u(-c)\right. \\
+ & \left.(-c)^{J} u a\right\}+\left\{c^{J} u a-a^{J} u c\right\} \in U+V,
\end{aligned}
$$

or $V A \subset U+V$. Similarly, $A(U+V) \subset V+V$. Therefore, $U+V$ is an ideal of $A$ and is dense in its closure $C$, a closed ideal of $A$. Thus, $U$ is dense in $C \cap S$. But $U$ is closed and so $C=U+\bar{V}$, the latter is the closure of $V$.

Now if $B A$ is dense in $B$ then given $b \in B$ there exists a net $b_{\alpha} a_{\alpha} \rightarrow b$ and so $b_{\alpha}(1 / 2) a_{\alpha} \rightarrow 1 / 2 b$ and $(1 / 2) a_{\alpha}^{J} b_{\alpha}^{J} \rightarrow 1 / 2 b^{J}$. Thus,

$$
b_{\alpha}(1 / 2) a_{\alpha}+(1 / 2) a_{\alpha}^{J} b_{\alpha}^{J} \rightarrow 1 / 2\left(b+b^{J}\right) .
$$

Hence, $1 / 2\left(b+b^{J}\right) \in U$, or if $b \in B \cap S$ then $b \in U$.

We define

$$
U_{J}=\{x \mid x \circ u=\theta \text { for all } u \in U\}
$$

and prove

Lemma 8. $U_{J}=\mathscr{L}(U)$, and if $a \in \mathscr{L}(U), a=s+k, s \in S, k \in K$ then $s$ and $k$ are in $\mathscr{L}(U)$.

If $x \in U_{J}$ then by Lemma $1, u x+x u=\theta$ and $x u^{2}+u^{2} x=\theta$ for each $u \in U$. Thus, $2 x u^{2}=\theta$; that is $x \in \mathscr{L}(U)$. So, $U_{J} \subset \mathscr{L}(U)$. Now by [1], $\mathscr{L}(B)=B_{J}$. Therefore, using Theorem 1 , we have $U_{J} \subset B_{J}$. But, since by Theorem $4, U \subset B$ we have, $B_{J} \subset U_{J}$. Thus $B_{J}=U_{J}=\mathscr{L}(U)$.

Now if $a=s+k \in \mathscr{L}(U)$ then $a \in \mathscr{L}(B)$ and so for $b \in B$, $(s+k) b=\theta$ and also $b^{J}(s+k)=\theta$, the latter since $\mathscr{R}(B)=\mathscr{L}(B)$. Now the conclusion is immediate.

4. Topological rings with property $(Y)$. Civen and Yood, [1], 
assume as a hypothesis in part of their work that $\mathscr{L}(I) \neq(\theta)$ for any closed proper two-sided ideal $I$ of $A, A$ a semi-prime ring. We note that this implies that $A^{3}$ is dense in $A$. For letting $I=\overline{A^{3}}$, we have $x \in \mathscr{L}(I)$ implies that $x^{4}=\theta$, a contradiction to semi-primeness.

We define an analogous condition for Jordan ideals of $S$.

PROPERTY $(Y)$. A, a topological ring, has property $(Y)$ if, and only if, (i) $2 A=A$; (ii) $A$ has a proper continuous involution such that whenever the net $\left\{2 x_{\alpha}\right\} \rightarrow 0$ then $\left\{x_{\alpha}\right\} \rightarrow 0$; (iii) $A^{3}$ is dense in $A$; and (iv) a closed Jordan ideal, $U$, of $S$ has $\mathscr{L}(U)=(\theta)$ if, and only if, $U=S$.

We now show that for such rings the main theorems of $\S 3$ hold.

Theorem 6. Let $A$ be a topological ring with property $(Y)$. Then for any closed Jordan ideal $U \subset S, \mathscr{L}(B)+B$ is dense in $A$ ( $B$ as defined in $\S 1$ ).

Consider $(\mathscr{L}(B)+B) \cap S=W$. By Lemma $2, \quad W$ is a Jordan ideal of $S$ and as before $\mathscr{C}(W) \cap S=(\theta)$. Thus, $y^{J} y=\theta$ for all $y \in \mathscr{L}(W)$ or $\mathscr{L}(W)=(\theta)$. Now, $\mathscr{L}(\bar{W})=\mathscr{L}(W)$ and so, by hypothesis, $\bar{W}=S$. Now, by Theorem $2, \bar{H}=B+\mathscr{L}(B)$ is a right ideal and $\bar{H} \cap S=\bar{W}=S$. Thus, given $a \in A$ then $a+a^{J}$ and $a^{J} a \in \bar{H}$. Thus, $\left(a+a^{J}\right) a \in \bar{H}$. Now, let $a \in A, b \in B$ then $b_{\alpha}+d_{\alpha} \rightarrow a b+b a$ where $b_{\alpha} \in B$ and $d_{\alpha} \in(B)$. Therefore,

$$
\left(b_{\alpha}-b a\right)+d_{\alpha} \longrightarrow a b .
$$

But, $b_{\alpha}-b a \in B$ and so $a b \in \bar{H}$. Since, $\mathscr{L}(B)$ is a (two-sided) ideal we conclude that $\bar{H}$ is an ideal with $A \circ A \subseteq \bar{H}$. It is well-known that this implies that $A^{3} \sqsubseteq \bar{H}$ or that $H$ is dense in $A$.

Corollary 1. Let $A$ be a topological ring with property ( $Y$ ). Then $I$, a closed two-sided ideal of $A$, with $\mathscr{L}(I)=(\theta)$, implies that $I=A$.

Note that in the last proof if one replaces $\bar{H}$ by $I$ one concludes that $\mathscr{L}(I)=(\theta)$ implies that $A^{3} \cong I$ and hence the desired conclusion.

Observing that this last corollary is the basis for much of the argument in $\S 3$, we conclude that

CoRollary 2. Let $A$ be a topological ring with property ( $Y$ ) then the conclusions of Theorem 3 and Theorem 5 hold.

5. Applications. We reprove Herstein's result for Jordan ideals 
of $S$ in a simple ring with involution. It is that theorem which motivates this paper.

THEOREM 7. [Herstein] Let $A$ be a simple ring with involution and with characteristic different than 2 . Let $Z$, the center of $A$, be zero or $[A: Z]>4$ then $S$ is simple Jordan.

By Lemma 2, $B$ is a right ideal and $S B \subset B$. By Herstein [2], under these hypothesis, the subring generated by $S$ is $A$ and so $B$ is a two-sided ideal. Now if $B=(\theta)$ then, by Lemma 2, $U \circ U=(\theta)$ and so $u S u=(\theta)$ for each $u \in U$. Now, as stated before, we have $U=(\theta)$. So, let $B=A$. Then for all $a, b \in A$ we have $(a, b)_{J} \in U$. Thus in particular if $s \in S$ then $(a s, b)_{J}=A s A \cap S \subset U$ and so $U=S$.

In the literature on algebras there is interest as to when the cone $P$, generated in $b b^{J}, b \in A$, is such that $P-P$ is dense in $S$. Here, we have not interested ourselves in algebras but rather in annihilator rings and we prove several results related to additive subgroups contained in $P-P$, the subgroup generated by the elements $b b^{J}, b \in A$. Note, that $S \circ S,[S, K]$ and $K \circ K$ are all subsets of $P-P$.

THEOREM 8. Let $A$ be a topological ring with property $(Y)$ or an annihilator ring with proper continuous involution, $2 A=A$, and the property that whenever the net $2 x_{\alpha} \rightarrow 0$ then $x_{\alpha} \rightarrow 0$, then we conclude that $S \circ S$ is dense in $S$.

We note that $S_{J}=(\theta)$. For, by Lemma $8, S_{J}=\mathscr{L}(S)$, and $x \in \mathscr{L}(S)$ implies that $x s=\theta$ for any $s \in S$ or $x^{J} x x^{J} x=\theta$ which yields the conclusion. Now, let $t \in \mathscr{L}(S \circ S) \cap S$. Then, since $S \circ S$ is a Jordan ideal of $S$, by Theorem 2 , we have that $\mathscr{L}(S \circ S)$ is a twosided ideal. Thus, for every $s \in S$

$$
t s+s t \in(S \circ S) \cap \mathscr{L}(S \circ S),
$$

which is $(\theta)$ by Lemma 5 and Theorem 1. Thus, $t \in S_{J}=(\theta)$ or $\mathscr{L}(S \circ S) \cap S=(\theta)$, and so $\mathscr{L}(S \circ S)=(\theta)$. Therefore

$$
C=\overline{S \circ S}+\left\{u a-a^{J} u \mid u \in \overline{S \circ S}\right\}
$$

is a two-sided ideal of $A$ and $\mathscr{L}(C)=\mathscr{L}(\bar{C}) \subset \mathscr{L}(\overline{S \circ S)}=\mathscr{L}(S \circ S)=$ $(\theta)$. Thus, $\bar{C}$ is dense in $A$. So, the symmetric part of $\bar{C}$; namely, $\overline{S \circ S}=S$ and we are done.

One might also consider the Jordan ideal $K \circ K+[S, K]=W$. If $t \in \mathscr{L}(W) \cap K$ then since

$$
t a+a^{J} t^{J} \in K \circ K+[S, K] \subset \bar{W}
$$


we have $t \in \mathscr{L}(W) \cap B_{\bar{W}}=(\theta)$, by Theorems 1 and 2. Using Lemma 6, we have $\mathscr{L}(W) \subset S$. $\mathscr{L}(W)$ being a two-sided ideal, implies that for all $a, b \in A, c \in \mathscr{L}(W)$ that $c a-a^{J} c=\theta$ and so $c a b-(a b)^{J} c=$ $c(a b-b a)=\theta$. Thus, in particular, $c(k l-l k)=\theta$ for all $k, l \in K$. Hence, $2 c k l=c(k l-l k)+c(k l+l k)=\theta$ or $c k^{2}=\theta$ for all $k \in K$, $c \in \mathscr{L}(W)$; that is, $c K=\theta$. Thus, $c \in Z$, the center of $A$ and if the latter is $(\theta)$ we conclude that $W$ is dense in $S$. Thus we have proved

TheOREM 9. Let $A$ be as in Theorem 8 and with zero center then $K \circ K+[S, K]$ is dense in $S$.

We are interested next in results concerning both minimal and minimal closed Jordan ideals of $S$ for rings $A$ of the previous sections.

THEOREM 10. Let $U$ be a minimal Jordan ideal of $S$ in a ring $A$ of $\S 2$. Then $U$ is the intersection of $S$ with a simple two-sided ideal.

Consider $T=\{t \in U \mid[t, K] \subset U\}$. As $U \neq(\theta)$ by assumption we are guaranteed by Lemmas 1 and 2 that $T \neq(\theta)$. Now, $t \in T$ then $t \circ s \in T$ for all $s \in S$ as $t \circ s \in U$ and $[t \circ s, K]=t \circ[s, K]+[t, K] \circ s$; that is, $T$ is a Jordan ideal of $S$. By minimality, $T=U$ or $[U, K] \subset U$. Thus, forming $V$, the additive subgroup generated by the set $\left\{(u, a)_{L} \mid u \in U, a \in A\right\}$ we have $U+V=C$ is a two-sided ideal of $A$. The latter is the same argument as in Theorem 5. Now, let $I \neq(\theta)$ be a two-sided ideal of $C$. Then, $H=C I C$ is a nonzero twosided ideal of $A, H \subset I$ (the latter uses the fact that the involution is proper). Thus, $H \cap S=U$. Therefore, for all $u \in U, a \in A$ we have $(u, a)_{L} \in H$ or $C \subset H$, completing the argument.

Definition. A nonzero closed Jordan ideal $U$, of $S$ is called minimal closed if, and only if, $H$ a closed Jordan ideal of $S, H \subseteq U$, implies $H=U$ or $H=(\theta)$.

THEOREM 11. Let $A$ be a topological ring with property $(Y)$ or an annihilator ring of $\S 3$, then there exists a one-to-one correspondence between the minimal closed Jordan ideals of $S$, and the minimal closed (hence topologically simple) two-sided ideals of $A$.

Let $U$ be a minimal closed Jordan ideal of $S$. Theorem 5 says that $I=U+\bar{V}$ is a closed two sided ideal of $A$. Let $H \neq(\theta)$ be a closed ideal of $A, H \subseteq I$. Then $H \cap S=U$. Thus, $u a-a^{J} u \in H$ for all $u \in U, a \in A$. That is $V \subset H$ and hence $U+\bar{V}=I \leqq H$; that is; $I$ is minimal closed two-sided. However, $A$ is semi-prime and so 
minimal-closed is equivalent to topologically simple. Thus, if we begin with $I$ topologically simple $\left(I=I^{J}\right.$ since the involution is proper) then we are interested in $I \cap S$. Let $U \neq(\theta)$ be a closed Jordan ideal of $S, U \subseteq I \cap S$. Then $U+\bar{V}$ is a closed ideal of $A$, contained in $I$. Thus, $U+\bar{V}=I$, or $I \cap S=U$ implying minimality. The one-to-one correspondence is clear.

We observe next that a topological sum of minimal closed Jordan ideals is a direct topological sum in the sense of Richart, [4] p. 46, (the generalization to Jordan ideals being the obvious). Let $U_{1}$, and $U_{2}$ be minimal closed Jordan ideals. $U_{1} \neq U_{2}$ implies that $U_{1} \cap U_{2}=(\theta)$. Thus $U_{1} \circ U_{2}=(\theta)$ or $U_{2} \in\left(U_{1}\right)_{J}=\mathscr{L}\left(U_{1}\right)=\mathscr{R}\left(U_{1}\right)$. Thus, $U_{1} U_{2}=$ $U_{2} U_{1}=(\theta)$. Now, let $U_{0}$ be a minimal closed Jordan ideal and $W$ the algebraic sum of the other minimal closed Jordan ideals. Then $x \in W$ implies $x=u_{1}+\cdots+u_{n}, u_{i} \in U_{i}$ where $U_{0} u_{i}=(\theta)$. Thus, $U_{0} W=(\theta)$ and so, $U_{0} \bar{W}=(\theta)$. Now, $U_{0} \cap \bar{W}$ is a closed Jordan ideal contained in $U_{0}$. If not zero, then $U_{0} \in \bar{W} \cap \mathscr{L}(\bar{W})=(\theta)$, the latter by Theorem 1 and Lemma 5. This is not possible. Thus, $U_{0} \cap \bar{W}=(\theta)$.

We make use of this to say something about the decomposition of $A$.

THEOREM 12. Let $A$ be a ring as described in paragraph 4. Assume that $S$ is a topological direct sum of its minimal closed Jordan ideals $\left\{U_{\alpha}\right\}$ then $A$ is a topological direct sum of minimal closed twosided ideals $\left\{I_{\alpha}\right\}$ where $I_{\alpha}=U_{\alpha}+\overline{\left[U_{\alpha}, U_{\alpha}\right]+\left(U_{\alpha} \circ K_{\alpha}\right)}, K_{\alpha}=I_{\alpha} \cap K$.

Let $x \in A$ then $x x^{J}$ and $x+x^{J} \in S$, and hence by Theorem 10, are in the topological direct sum, $T$, of the minimal closed ideals. Thus, $x^{2} \in T$ for all $x \in A$, and so $A^{3} \subset T$. Therefore, $A=\bar{A}^{3}$ implies that $A=T$.

Now, $A=\overline{\oplus \sum I_{\alpha}}$, where $I_{\alpha}=U_{\alpha}+\bar{V}_{\alpha}, U_{\alpha}=S \cap I_{\alpha}$ and $\bar{V}_{\alpha}=$ $K \cap I_{\alpha}$. Let $x_{\beta} \rightarrow a, x_{\beta}=\sum y_{\gamma \beta} \in \sum I_{\gamma}$. Then, $u_{\alpha} a-a^{J} u_{\alpha} \in V_{\alpha}, u_{\alpha} \in U_{c}$ is such that

$$
u_{\alpha} y_{\alpha \beta}-y_{\alpha \beta}^{J} u_{\alpha}=u_{\alpha} x_{\beta}-x_{\beta}^{J} u_{\alpha} \longrightarrow u_{\alpha} a-a^{J} u_{\alpha} .
$$

Therefore, $u_{\alpha} a-a^{J} u_{\alpha} \in \overline{\left.U_{\alpha}, U_{\alpha}\right]+U_{\alpha} \circ K_{\alpha}}$ which yields the desired conclusion.

CoRollary 3. Hypothesis of Theorem 12, then every closed Jordan ideal $U \neq(\theta)$ contains a minimal closed Jordan ideal. Moreover, if $U_{\alpha}$ is a minimal closed Jordan ideal then either $U_{\alpha} \subseteq U$ or $U_{\alpha} \in \mathscr{L}(U)$.

We see that for each $U_{\alpha}, U_{\alpha} \cap U$ is either $(\theta)$ or $U_{\alpha}$. If the former, then $x \in U_{\alpha}$ implies that $x \in U_{J}=\mathscr{L}(U)$. However, if $U_{\alpha} \cap U=(\theta)$ for all $\alpha$, then $S \in \mathscr{L}(U)$ and so $U=(\theta)$, a contradiction. 
We are interested in saying something about idempotents; in particular, minimal symmetric idempotents (as defined in Rickart [4], Chapter II). If $I$ is a minimal left ideal in a ring with proper involution then $I=A e$, where $e=e^{2} \in S$ (Rickart [4], p. 261).

Lemma 7. Let $U$ be a Jordan ideal in a ring $A$ of $\S 2$. Let $e \neq \theta$ be a minimal symmetric idempotent then either $e \in U \cap B$ or $e \in \mathscr{L}(U)$.

Let $U \neq(\theta)$ be given and let $B$ be as defined in $\S 2$. Then, $e B \subset B$ and $e B \subset e A$. Therefore, if $e \notin \mathscr{L}(B)=\mathscr{L}(U)$ then $e B=e A$. However, $e \in e A$ and thus, $e \in e B \subset B$. So, $(e, 1 / 2 e)_{J}=e \in U$.

THEOREm 13. Let $A$ be as in $\S 2$. Then, if $U$ is a Jordan ideal of $S$ and if $e \neq \theta, e=e^{2} \in U$ then the homogeneous component containing $e$ (for definition see Jacobson [3], chapter 4) interected with $S$ is contained in $U$.

We note that if $e \in U$ then by Lemma 5, $e \in B$. Also $(x e, y)_{J}=$ $\left(x e^{2}, y\right)_{J}=\left((x, e)_{J}, y\right)_{J}+\left(e,-x^{J} e y\right)_{J}$. Hence, $(x e, y)_{J}=x e y+y^{J} e x^{J} \in U$, or $A e A \cap S \subset U$. But, the homogeneous component of $A$ containing $e$ is simple and so the desired result.

CoRollary 4. Let $A$ be a primitive ring with proper involution, $2 A=A, 2 x=\theta$ implies $x=\theta$, and with nonzero socle, $\zeta$, then every nonzero Jordan ideal $U \subset S$ contains $\zeta \cap S$.

Let $U \neq(\theta)$ be given. Then, $B \neq(\theta)$ and $\mathscr{L}(B)$ is a two-sided ideal. Now, primitive implies prime and thus $\mathscr{f}(B)=(\theta)$. Thus, every symmetric idempotent of Lemma 7 is in $B$. Let $x \in \zeta \cap S x=$ $\sum_{i=1}^{n} e_{i} a_{i}=\sum_{i=1}^{n} a_{i}^{J} e_{i}$. Thus, $x=\sum_{i=1}^{n}\left(e_{i},(1 / 2) a_{i}\right)_{J} \in U$.

Corollary 5. Let $A$ be a topological ring which is primitive and has dense socle. Let $A$ have properties (i) and (ii) of property $(Y)$. Then, $S$ is minimal closed.

Let $U \neq(\theta)$ be a closed Jordan ideal of $S$. Then, by Corollary 5, $U$ contains $\zeta \cap S$ and hence its closure which is $S$.

THEOREM 14. Let $A$ be a topological ring with properties (i) and (ii) (of property $(Y)$ ) and suppose that $A$ has dense socle than if $U$ is a maximal closed Jordan ideal of $S, U$ is the intersection of $S$ with a closed two-sided ideal, I.

Let $U$ be as given. If $[U, K] \subset U$ then as before $U+\bar{V}=C$ has 
the desired property. Now, if $e \in U$ for all minimal symmetric idempotents then $e \in B$ as $e a+a^{J} e=e\left(e a+a^{J} e\right) e+e\left(-a-a^{J}\right) e \in U$ for all $a \in A$. Thus, $\zeta \cap S \subset U$ and hence $S \subset U$, a contradiction. Therefore there exists a minimal symmetric idempotent $f$ such that $f \notin U$. By Lemma $7, f \in \mathscr{L}(U)$. Now, $U+[U, K]$ is a Jordan ideal of $S$, and so by the previous remarks, we assume that $U+[U, K]$ is dense in $S$. Therefore, there exists a net $x_{\alpha} \in U+[U, K]$ such that $x_{\alpha} \rightarrow f$. Now, $x_{\alpha}=u_{\alpha}+\sum_{i=1}^{n} v_{\alpha_{i}} k_{\alpha_{i}}-k_{\alpha_{i}} v_{\alpha_{i}}$ and so $f x_{\alpha}=f u_{\alpha}+\sum_{i=1}^{n}\left(f v_{\alpha_{i}}\right) k_{\alpha_{i}}-$ $\left(f k_{\alpha_{i}}\right) v_{\alpha_{i}}=\theta$. Therefore, $f x_{\alpha} \rightarrow f^{2}=\theta$, a contradiction. Hence, $[U, K] \subset U$, and we are done.

6. An example. In $\S 2$ we showed that $B$ is a right ideal. We now construct an example of a ring with proper involution (indeed the ring is an integral domain) in which $B$ is not a two-sided ideal. Let $R$ be the set consisting of the polynomials in two commuting indeterminates over the complex numbers of the form

$$
i \tau\left(x^{1} y^{0}+x^{0} y^{1}\right)+u\left(x^{2} y^{0}+x^{0} y^{2}\right)+c x^{1} y^{1}+\sum_{i+j \geqq 3} a_{i J} x^{i} y^{j}
$$

where $\tau$ and $u$ are real, and where $c$ and $a_{i j}$ are complex numbers. Assume the usual addition and multiplication defined by

$$
\left(\sum b_{r s} x^{r} y^{s}\right)\left(\sum c_{k l} x^{k} y^{l}\right)=\sum b_{r s} c_{k l}^{(r+s)} x^{r+k} y^{s+1}
$$

where $c^{(n)}$ denotes conjugation $n$ times. One verifies that $R$ is a ring and that the mapping $\sum b_{r s} x^{r} y^{s} \stackrel{J}{\longrightarrow} \sum b_{r s}^{(r+s)} x^{s} y^{r}$ is an involution with

$$
S=\left(u\left(x^{2} y^{0}+x^{0} y^{2}\right)+c x^{1} y^{1}+\sum_{i+j \geqq 3} a_{i j} x^{i} y^{j} \mid \alpha_{j i}^{(i+j)}=a_{i j}\right\} \text {. }
$$

Letting

$$
\begin{gathered}
U=\left\{\sigma\left(x^{2} y^{1}+x^{1} y^{2}\right)+\tau(1+i)\left(x^{3} y^{1}+x^{1} y^{3}\right)+F x^{2} y^{2}+\sum_{i+j \geqq 5} u_{i j} x^{i} y^{j} \mid \sigma, \tau\right. \\
\text { real; } \left.F, u_{i j} \text { complex; and } u_{i j}^{(i+j)}=u_{i j}\right\},
\end{gathered}
$$

we see that if $u \in U, s \in S$ then $u s+s u$ has each term of degree at least 5 and so $U$ is a Jordan ideal of $S$. Now let $b=(1+i) x^{1} y^{1}$ then $b a+a^{J} b^{J}$ is in $U$ for all $a \in R$. Thus, $b \in B$. Setting $k=i\left(x^{1} y^{0}+x^{0} y^{1}\right)$ then one verifies that

$$
(k b) k+k^{J}(k b)^{J}=2 k b k=2(1-i)\left(x^{3} y^{1}+x^{1} y^{3}\right)+4(1-i) x^{2} y^{2}
$$

is not in $U$. Thus $B$ is not two-sided.

We note that if $B=B^{J}$ then $B$ is a two-sided ideal. Furthermore if $B$ is two-sided then 


$$
\left(a^{J} b, c^{J}\right)_{J}=\left(c b^{J}, a\right)_{J} \in U
$$

for all $a, c \in A$ and $b \in B$. Therefore, $A b^{J} \in B$. Thus, for example if $A$ has an identity then $B=B^{J}$. We now show that $B$ being twosided does not guarantee that $B=B^{J}$. Consider $A_{1}=\left\{\lambda\left(x^{1} y^{0}-x^{0} y^{1}\right)+\right.$ $C x^{2} y^{0}+D x^{0} y^{2}+F x^{1} y^{1}+\sum_{i+j \geqq 3} \alpha_{i j} x^{i} y^{j} \mid \lambda$ is real, $C, D, F$ and $\alpha_{i j}$ complex $\} ; \quad U=\left\{\sigma(1+i)\left(x^{3} y^{0}-x^{2} y^{1}\right)+\sigma(1-i)\left(x^{0} y^{3}-x^{1} y^{2}\right)+\sum_{i+j \geqq 4} \beta_{i j} x^{i} y^{j} \mid \sigma\right.$ is real, $\left.\beta_{i j}=\beta_{j i}^{(i+j)}\right\}$ and $b=(1+i) x^{2} y^{0}$. Assuming the previously defined multiplication one verifies that $B$ is two-sided, $b \in B$ but $b^{J} \notin B$.

The author wishes to express his deepest thanks to the Department of Mathematics, University of Oregon, for the cordiality shown him during his stay there at which time this manuscript was prepared, and particularly to Professor Bertram Yood for his many suggestions and kindness.

\section{REFERENCES}

1. P. Civen and B. Yood, Lie and Jordan structures in Banach algebras, Pacific J. Math. 15 (1965), 775-797.

2. I. N. Herstein, Lie and Jordan systems in simple rings with involution, Amer. J. Math. 78 (1956), 629-649.

3. N. Jacobson, Structure of rings, A. M. S. Coll. Publ. 37 (1956).

4. C. E. Rickart, General Theory of Banach Algebras, Van Nostrand, New York, 1960.

Received August 11, 1967.

UNIVERSITY OF DELAWARE 


\section{PACIFIC JOURNAL OF MATHEMATICS}

EDITORS

H. ROYDEN

Stanford University

Stanford, California

R. R. Phelps

University of Washington

Seattle, Washington 98105
J. DugundJI

Department of Mathematics

University of Southern California

Los Angeles, California 90007

\section{RICHARD ARENS}

University of California

Los Angeles, California 90024

\section{ASSOCIATE EDITORS}
E. F. BeCKENBACH
B. H. NeUmanN
F. WOLF
K. YOSIDA

\section{SUPPORTING INSTITUTIONS}

UNIVERSITY OF BRITISH COLUMBIA CALIFORNIA INSTITUTE OF TECHNOLOGY UNIVERSITY OF CALIFORNIA MONTANA STATE UNIVERSITY UNIVERSITY OF NEVADA NEW MEXICO STATE UNIVERSITY OREGON STATE UNIVERSITY UNIVERSITY OF OREGON OSAKA UNIVERSITY UNIVERSITY OF SOUTHERN CALIFORNIA
STANFORD UNIVERSITY UNIVERSITY OF TOKYO UNIVERSITY OF UTAH WASHINGTON STATE UNIVERSITY UNIVERSITY OF WASHINGTON

AMERICAN MATHEMATICAL SOCIETY CHEVRON RESEARCH CORPORATION TRW SYSTEMS NAVAL WEAPONS CENTER 


\section{Pacific Journal of Mathematics \\ Vol. 27, No. $1 \quad$ January, 1968}

Willard Ellis Baxter, On rings with proper involution ............... 1

Donald John Charles Bures, Tensor products of $W^{*}$-algebras........... 13

James Calvert, Integral inequalities involving second order derivatives . . . . 39

Edward Dewey Davis, Further remarks on ideals of the principal class.... 49

Le Baron O. Ferguson, Uniform approximation by polynomials with integral

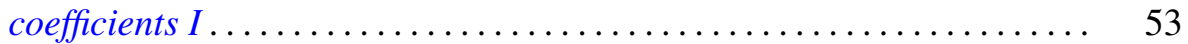

Francis James Flanigan, Algebraic geography: Varieties of structure

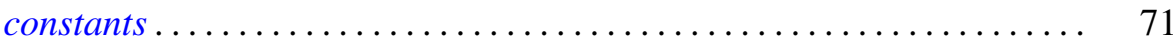

Denis Ragan Floyd, On QF -1 algebras ..................... 81

David Scott Geiger, Closed systems of functions and predicates ......... 95

Delma Joseph Hebert, Jr. and Howard E. Lacey, On supports of regular Borel measures ................................... 101

Martin Edward Price, On the variation of the Bernstein polynomials of a function of unbounded variation ........................ 119

Louise Arakelian Raphael, On a characterization of infinite complex matrices mapping the space of analytic sequences into itself........ 123

Louis Jackson Ratliff, Jr., A characterization of analytically unramified

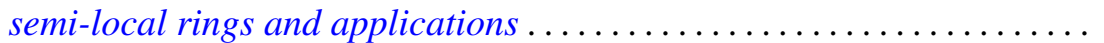

S. A. E. Sherif, A Tauberian relation between the Borel and the Lototsky transforms of series ................................ 145

Robert C. Sine, Geometric theory of a single Markov operator .......... 155

Armond E. Spencer, Maximal nonnormal chains in finite groups......... 167

Li Pi Su, Algebraic properties of certain rings of continuous functions .... 175

G. P. Szegô, A theorem of Rolle's type in $E^{n}$ for functions of the class $C^{1} \ldots 193$

Giovanni Viglino, A co-topological application to minimal spaces ........ 197

B. R. Wenner, Dimension on boundaries of $\varepsilon$-spheres ............... 201 